\title{
Iron abundance in the prototype PG 1159 star, GW Vir pulsator PG 1159-035, and related objects ${ }^{\star}$
}

\author{
K. Werner ${ }^{1}$, T. Rauch ${ }^{1}$, J. W. Kruk ${ }^{2}$, and R. L. Kurucz ${ }^{3}$ \\ 1 Institute for Astronomy and Astrophysics, Kepler Center for Astro and Particle Physics, Eberhard Karls Universität Tübingen, \\ Sand 1, 72076 Tübingen, Germany \\ e-mail: werner@astro.uni-tuebingen.de \\ 2 NASA Goddard Space Flight Center, Greenbelt, MD 20771, USA \\ ${ }^{3}$ Harvard-Smithsonian Center for Astrophysics, 60 Garden Street, Cambridge, MA 02138, USA
}

Received 31 March 2011 / Accepted 3 May 2011

\section{ABSTRACT}

\begin{abstract}
We performed an iron abundance determination of the hot, hydrogen deficient post-AGB star PG 1159-035, which is the prototype of the PG 1159 spectral class and the GW Vir pulsators, and of two related objects (PG 1520+525, PG 1144+005), based on the first detection of Fe vIII lines in stellar photospheres. In another PG 1159 star, PG 1424+535, we detect Fe vII lines. In all four stars, each within $T_{\text {eff }}=110000-150000 \mathrm{~K}$, we find a solar iron abundance. This result agrees with our recent abundance analysis of the hottest PG 1159 stars $\left(T_{\text {eff }}=150000-200000 \mathrm{~K}\right)$ that exhibit Fe x lines. On the whole, we find that the PG 1159 stars are not significantly iron deficient, in contrast to previous notions.
\end{abstract}

Key words. stars: abundances - stars: atmospheres - stars: evolution - stars: AGB and post-AGB - white dwarfs

\section{Introduction}

PG 1159 stars are hot hydrogen deficient post-AGB stars (Werner \& Herwig 2006). In the Hertzsprung-Russell diagram, they cover a region comprising the hottest central stars of planetary nebulae and white dwarfs $\left(T_{\text {eff }}=75000-200000 \mathrm{~K}, \log g=\right.$ 5.5-8). Their $\mathrm{H}$ deficiency is most probably the result of a late $\mathrm{He}$-shell flash. Their envelopes are mainly composed of $\mathrm{He}, \mathrm{C}$, and $\mathrm{O}$, with rather diverse abundance patterns $(\mathrm{He}=0.30-0.85$, $\mathrm{C}=0.13-0.60, \mathrm{O}=0.02-0.20$, mass fractions).

The prototype PG 1159-035 (=GW Vir) was discovered in the Palomar Green survey (Wesemael et al. 1985). Subsequently it was found that the star is variable (McGraw et al. 1979) and it also became the prototype of the GW Vir stars, which are non-radial multimode g-mode pulsators. Besides the Sun, PG 1159-035 is probably the star that is best studied with asteroseismic methods (Costa et al. 2008). One of the key questions related to these pulsators concerns the driving mechanism, because the instability strip occupied by them is not "pure" like the ZZ Ceti strip, meaning that it also contains non-variable PG 1159 stars.

The primary pulsation driver is cyclic ionisation of $\mathrm{C}$ and O (Starrfield et al. 1984). The location of the instability strip is "fuzzy", because the red and blue edges of the strip depend on the $\mathrm{He} / \mathrm{C} / \mathrm{O}$ abundance ratio in the driving region; too high an He abundance poisons pulsations (Quirion et al. 2007). Another

* Based on observations made with the NASA-CNES-CSA Far Ultraviolet Spectroscopic Explorer. FUSE was operated for NASA by the Johns Hopkins University under NASA contract NAS5-32985. Based on observations with the NASA/ESA Hubble Space Telescope, obtained at the Space Telescope Science Institute, which is operated by the Association of Universities for Research in Astronomy, Inc., under NASA contract NAS5-26666. species that supports pulsation driving is iron, therefore, a subsolar iron abundance would narrow the instability strip.

PG 1159-035 $\left(T_{\text {eff }}=140000 \mathrm{~K}, \log g=7\right)$, together with a near spectroscopic twin, the non-pulsator PG $1520+525\left(T_{\text {eff }}=\right.$ $150000 \mathrm{~K}, \log g=7.5$ ), potentially defines the blue edge of the GW Vir strip, provided their envelope chemical composition is similar. Considerable effort was put into spectroscopic analyses to derive temperature, gravity, and composition of these twin stars. One remaining open question is the iron abundance. For some PG 1159 stars, including the twins, there were claims of iron deficiency (Jahn et al. 2007).

Spectroscopically, the iron abundance in PG 1159 stars is difficult to assess. Hitherto, the main tool were ultraviolet Fe vII lines, well known from observations of hot hydrogen-rich central stars of planetary nebulae. Because of the high effective temperatures, these lines are predicted to be rather weak or even undetectable in PG 1159 stars. The previously mentioned $\mathrm{Fe}$ deficiency was based on not detecting Fe vII lines. UV lines from higher ionisation stages of iron were unknown until recently, when Fe $x$ lines were detected in five of the very hottest $\left(T_{\text {eff }} \geq 150000 \mathrm{~K}\right)$ PG 1159 stars (Werner et al. 2010). A solar iron abundance was derived.

In this paper, we announce the detection of Fe vIII lines in FUSE spectra of three medium-hot $\left(T_{\text {eff }}=140000-150000 \mathrm{~K}\right)$ PG 1159 stars, including the prototype and its twin. They serve as a tool for determining the iron abundance, closing the gap between the coolest $\left(T_{\text {eff }} \lesssim 140000 \mathrm{~K}\right.$ ) PG 1159 stars, where we should be able to detect Fe viI, and the very hottest objects exhibiting Fe $\mathrm{x}$. For the first time, we present an iron abundance determination of PG 1159-035. We carefully re-assess archival FUSE and HST spectra of PG 1159-035 to look for weak, previously undetected Fe vII lines. This search is extended to the cooler object PG $1424+535\left(T_{\text {eff }}=110000 \mathrm{~K}, \log g=7\right)$, where the non-detection of these lines would mean a Fe deficiency of 


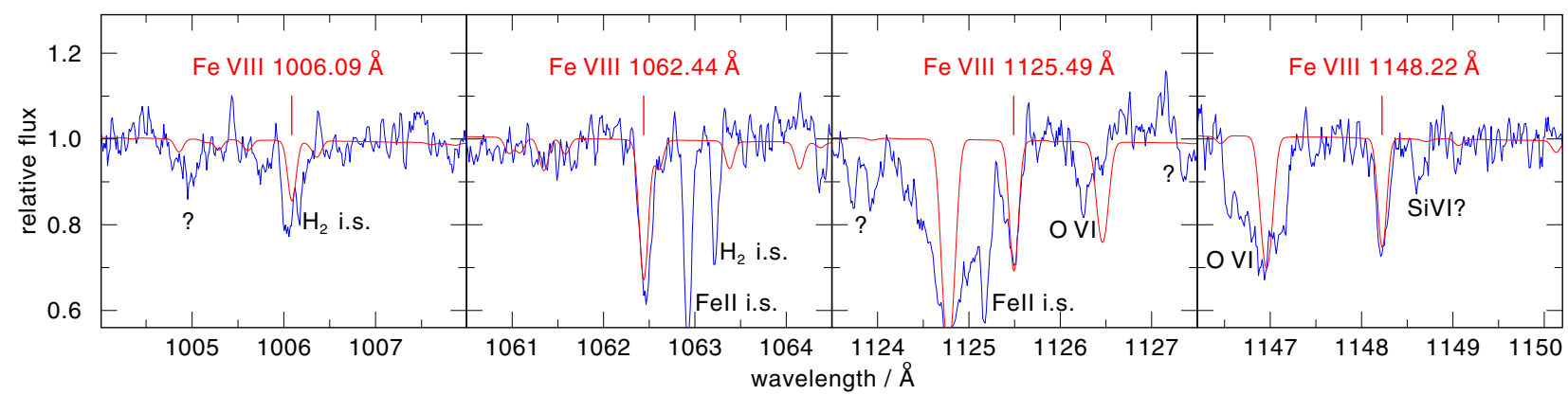

Fig. 1. Fe VIII lines detected in PG 1159-035. Overplotted is the final model with solar iron abundance (model parameters: $T_{\text {eff }}=140000 \mathrm{~K}, \log g=$ $7, \mathrm{He} / \mathrm{C} / \mathrm{O} / \mathrm{Ne}=0.32 / 0.48 / 0.17 / 0.02$; mass fractions).

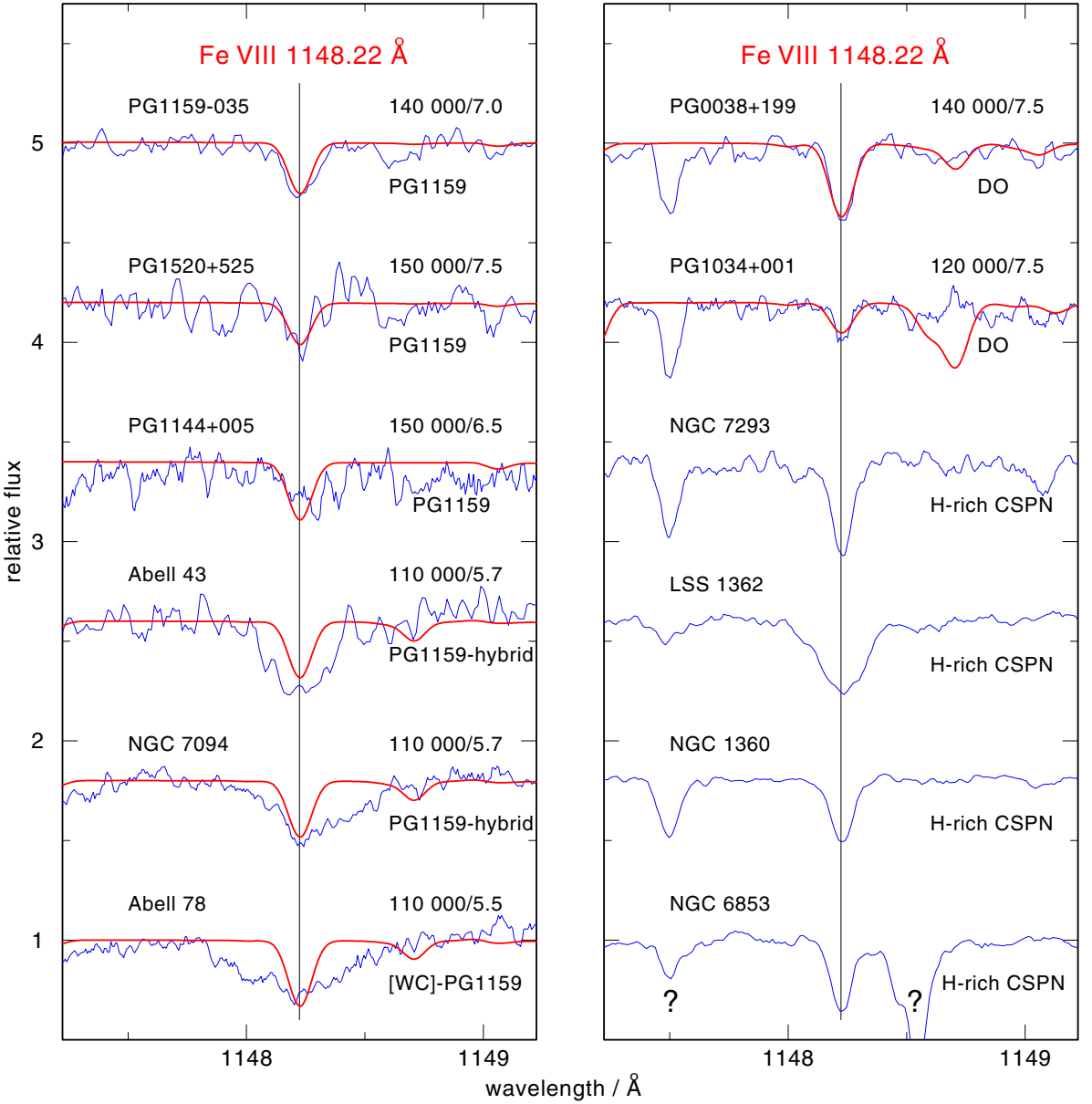

Fig. 2. The Fe VIII $\lambda 1148.22 \AA$ line in PG 1159 stars and other objects. Left panel, from top: three PG 1159 stars, two PG 1159-hybrids, and a [WC]-PG 1159 transition object. Right panel: two hot DOs and four H-rich central stars. Depicted numbers denote temperature and gravity of the overplotted (red) models. They have solar iron abundance except for the two DOs where Fe is ten times solar. one dex (Reiff et al. 2008). We also report on Fe viII lines in other hot $\mathrm{H}$ deficient and $\mathrm{H}$ rich post-AGB stars.

In the following section, we present the detection of Fe vIII lines in PG 1159-035 and other objects (Sect. 2). Then we describe our model atmospheres (Sect.3) and the spectroscopic iron abundance analysis of four PG 1159 stars (Sect. 4), and we conclude in Sect. 5.

\section{Observations and line identifications}

Recently, Landi \& Young (2010) have been able to identify four Fe vIII coronal emission lines in the $\lambda 1000-1200 \AA$ region of the quiet Sun, in spectra obtained with the SOHO/SUMER instrument. This prompted us to look for accordingly photospheric lines in FUSE spectra of PG 1159 stars. All four Fe vIII lines are present in the prototype PG 1159-035 (Fig. 1). We find Fe VIII lines in two more PG 1159 stars and in several other hot (pre-) white dwarfs as well. From these objects we display the region around Fe vIII $\lambda$ 1148.22 $\AA$ in Fig. 2.

The two other PG 1159 stars have slightly higher temperatures than the prototype $\left(\mathrm{PG} 1520+525: T_{\mathrm{eff}}=150000 \mathrm{~K}\right.$, $\log g=7.5 ;$ PG 1144+005: $T_{\text {eff }}=150000 \mathrm{~K}, \log g=6.5$ ). Different PG 1159 stars from which FUSE spectra exist are obviously too hot or too cool to exhibit Fe vIII lines. In particular, these are the hottest ones exhibiting Fe $\mathrm{x}$ lines mentioned in the introduction $\left(T_{\mathrm{eff}} \geq 150000 \mathrm{~K}\right)$, and the cooler object PG $1424+535\left(T_{\text {eff }}=110000 \mathrm{~K}\right)$ that will be discussed below (Sect. 4.2).

The central stars Abell 43 and NGC 7094 are hybridPG 1159 stars (i.e. exhibiting H-Balmer lines), and Abell 78 is a [WC]-PG 1159 transition object. They all have low surface gravity, and the extraordinary wide profiles indicate that the Fe vIII 
K. Werner et al.: Iron abundance in PG 1159-035 and related objects

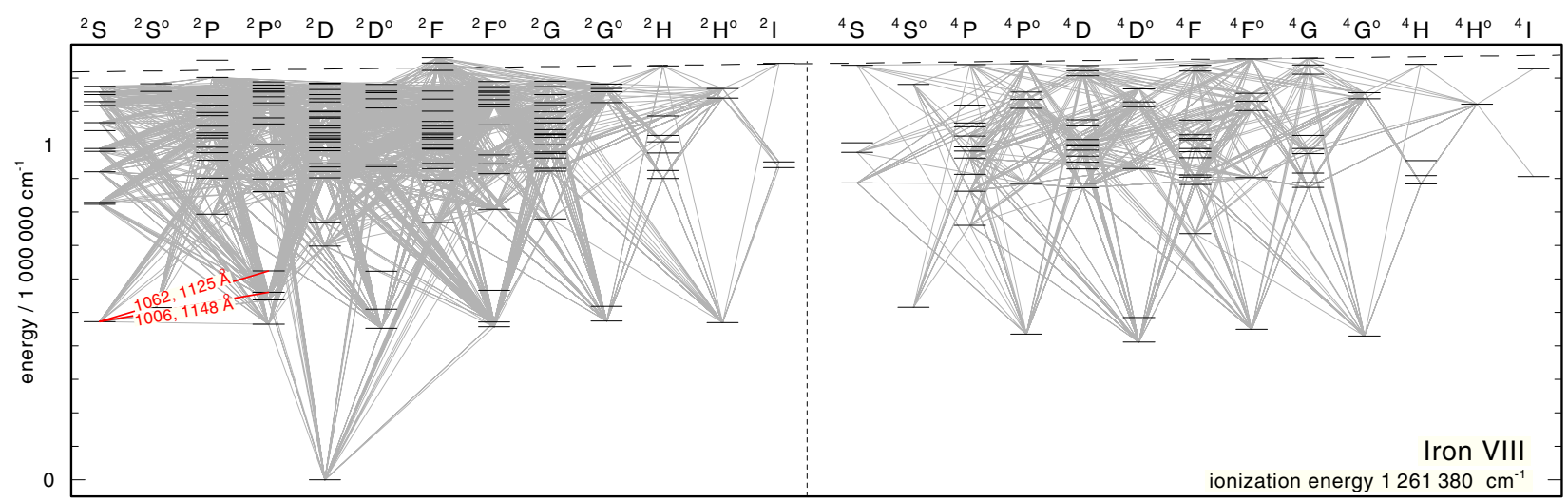

Fig. 3. Grotrian diagram of Fe vIII. For clarity, it is drawn from Opacity Project (OP) data that represent a small subset of the Kurucz dataset utilised in our computations. The OP level energies differ from Kurucz values. The transitions giving rise to the observed lines are indicated.

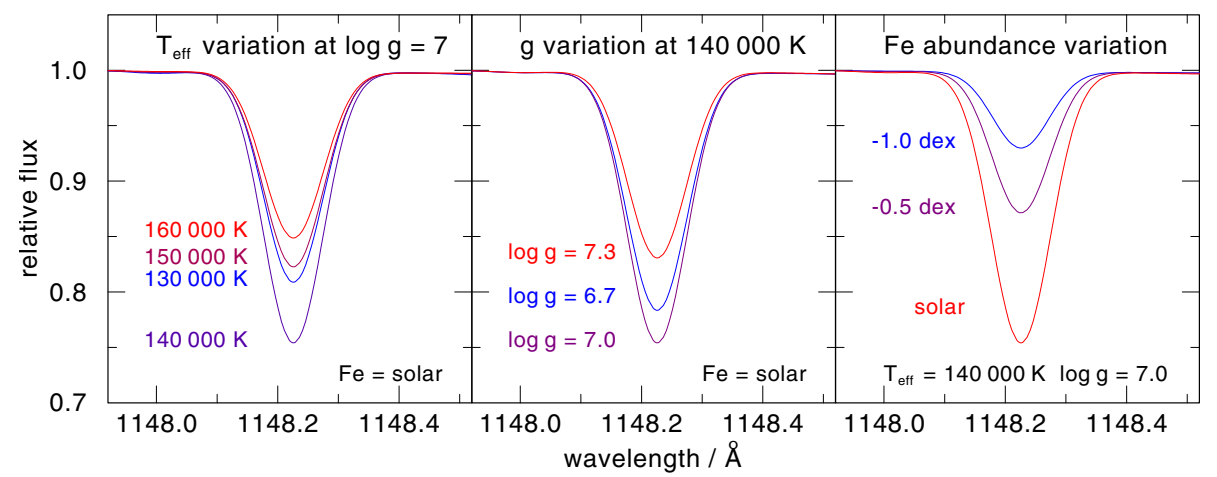

Fig. 4. Effects of model parameter variations on the Fe VIII $\lambda 1148.22 \AA$ line profile. The line strength is maximum at the parameters of PG $1159-035\left(T_{\text {eff }}=140000 \mathrm{~K}\right.$, $\log g=7)$; other abundances are $\mathrm{He} / \mathrm{C} / \mathrm{O} / \mathrm{Ne}=$ $0.32 / 0.48 / 0.17 / 0.02$. lines are strongly affected by a stellar wind. The low surface gravity also favours the appearance of Fe viII although $T_{\text {eff }}$ of these stars is relatively low $(\approx 110000 \mathrm{~K})$.

Two of the hottest known DO white dwarfs, PG0038+199 with $T_{\text {eff }}=115000 \mathrm{~K}$ and PG1034+001 with $100000 \mathrm{~K}$ (Dreizler \& Werner 1996), display Fe vIII lines. Comparison with preliminary model calculations indicates a necessary upward revision of the temperatures by $\approx 20000 \mathrm{~K}$ in order to reproduce these lines. We detect Fe vIII in neither KPD 0005+5106, the hottest DO (200000 K, Wassermann et al. 2010), nor in cooler DOs like RE J0503-289 (70000 K, Dreizler \& Werner 1996).

We also find Fe viII lines in several hydrogen-rich central stars. Four very prominent examples are displayed in Fig. 2: NGC $7293\left(T_{\text {eff }}=120000 \mathrm{~K}, \log g=6.3\right), \operatorname{LSS} 1362\left(T_{\mathrm{eff}}=\right.$ $114000 \mathrm{~K}, \log g=5.7)$, NGC $1360\left(T_{\mathrm{eff}}=97000 \mathrm{~K}, \log g=\right.$ $5.3)$, NGC $6853\left(T_{\text {eff }}=126000 \mathrm{~K}, \log g=6.5\right)$; the parameters are from Hoffmann et al. (2005).

\section{Model atmospheres and synthetic spectra}

For our analysis we use a grid of line-blanketed non-LTE model atmospheres, which is described in detail in Werner et al. (2004). In essence, the models include the main photospheric constituents, namely $\mathrm{He}, \mathrm{C}, \mathrm{O}, \mathrm{Ne}$, and occasionally $\mathrm{H}$. NLTE line formation iterations for the iron population densities were computed on these model structures, i.e., keeping fixed temperature and density structure. For details on the used iron model atom, see Wassermann et al. (2010). We employ new versions of iron datasets (Kurucz 2009) ${ }^{1}$. They include many more levels and lines, in particular the four Fe vIII lines discussed in this paper.

Properties of the newly detected Fe vIII lines are listed in Table 1. They all arise from the same lower level. We specify

\footnotetext{
${ }^{1}$ http://kurucz.harvard.edu/atoms.html
}

Table 1. Wavelengths and oscillator strengths $f_{i j}$ of Fe VIII lines.

\begin{tabular}{llll}
\hline \hline Line & $\lambda_{\text {Kurucz }} / \AA$ & $\lambda_{\text {Landi }} / \AA$ & $f_{i j}$ \\
\hline $4 \mathrm{~s}^{2} \mathrm{~S}_{1 / 2}-3 \mathrm{~d}^{2}{ }^{2} \mathrm{P}_{1 / 2}^{\mathrm{o}}$ & 1006.087 & 1006.015 & 0.0169 \\
$4 \mathrm{~s}^{2} \mathrm{~S}_{1 / 2}-4 \mathrm{p}{ }^{2} \mathrm{P}_{3 / 2}^{\mathrm{o}}$ & 1062.440 & $1062.463^{1}$ & 0.401 \\
$4 \mathrm{~s}^{2} \mathrm{~S}_{1 / 2}-4 \mathrm{p}{ }^{2} \mathrm{P}_{1 / 2}^{\mathrm{o}}$ & 1125.492 & 1125.546 & 0.216 \\
$4 \mathrm{~s}{ }^{2} \mathrm{~S}_{1 / 2}-3 \mathrm{~d}^{2}{ }^{2} \mathrm{P}_{3 / 2}^{\mathrm{o}}$ & 1148.224 & 1148.223 & 0.0828 \\
\hline
\end{tabular}

Notes. ${ }^{(1)}$ Mean value from two measurements.

the Kurucz wavelengths, as well as those measured by Landi \& Young (2010). The differences are all smaller than $0.1 \AA$. The largest deviation $(0.072 \AA)$ is shown by the $1006 \AA$ line. The Kurucz wavelengths should be more accurate than the measured wavelengths since the energy levels involved were determined from more than one line. We also list the $f$-values from the Kurucz data. A simplified Grotrian diagram indicating the observed line transitions is shown in Fig. 3.

We computed a small model grid in order to study the dependence of the Fe vIII lines on $T_{\text {eff }}, \log g$, and Fe abundance. The result for $\lambda 1148 \AA$ is displayed in Fig. 4, and the other lines behave similarly. It turns out that effective temperature and gravity of PG 1159-035 are the most favourable for the detection of Fe VIII. It also explains why Fe vIII lines are not seen in objects that are much cooler or hotter.

\section{Iron abundance analysis}

\section{1. $P G 1159-035$}

Figure 1 shows Fe vIII lines profiles computed from a solar Fe abundance model for PG 1159-035 compared to the 

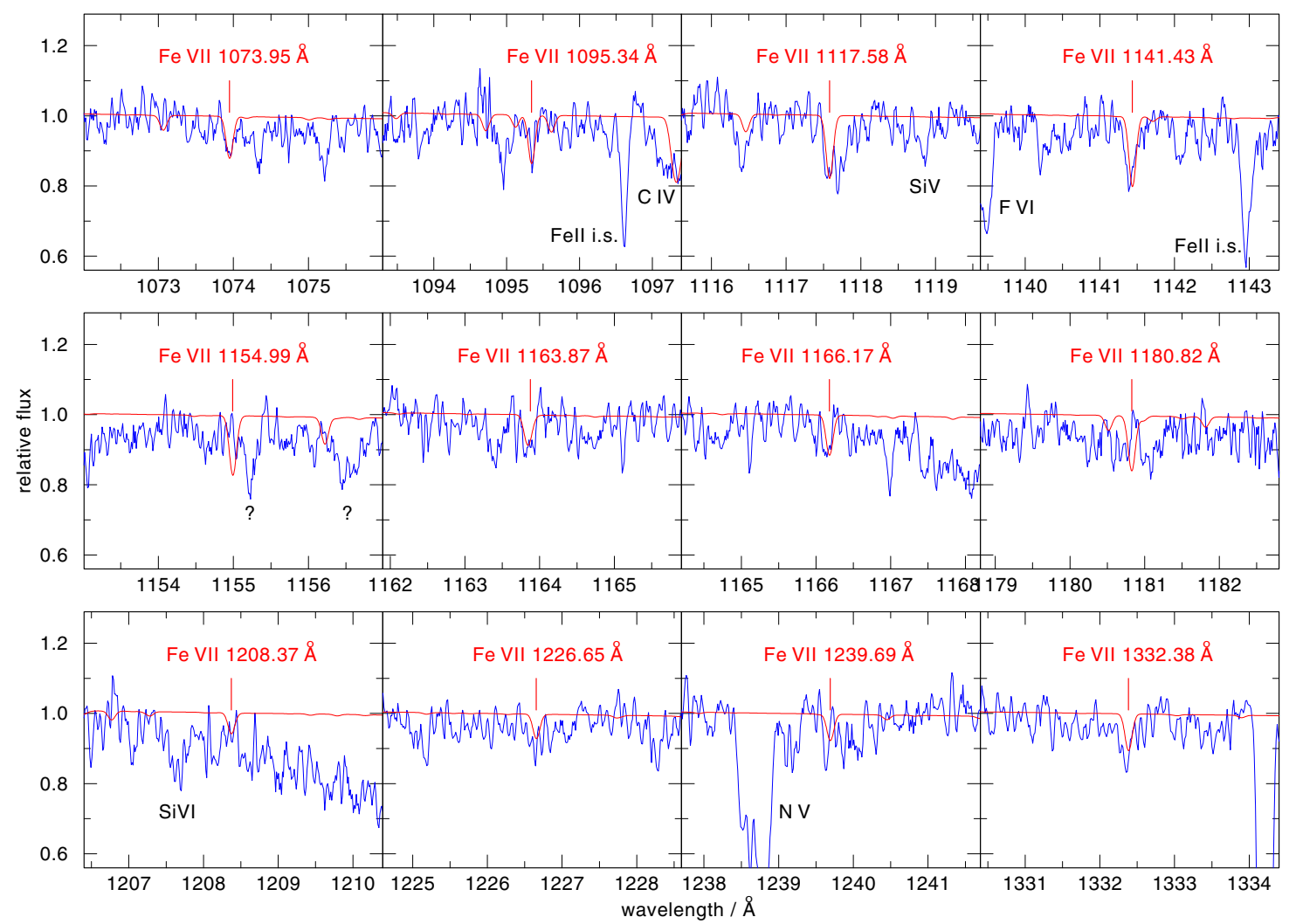

Fig. 5. Fe vII lines in PG 1159-035. Overplotted is the final model with solar iron abundance (model parameters like in Fig. 1). FUSE data are used for $\lambda<1200 \AA$ and HST data otherwise.

observation. The fit is satisfactory, and a comparison with the Fe variation shown in the right panel of Fig. 4 clearly rules out a significant iron deficiency.

In contrast, we previously decided there is an iron deficiency of $>0.7$ dex from not detecting Fe viI lines (Jahn et al. 2007), so we need to address this question again here. A close inspection of the FUSE and HST spectra reveals a number of weak Fe vII lines (Fig. 5), which are fitted by our solar Fe abundance model.

The reason we rejected the Fe vII detection in our previous work was the apparent absence of the two strong predicted lines at $\lambda \lambda 1154.99$ and $1180.82 \AA$ in the FUSE data. The cause of the non-detection remains unclear. In particular, we carefully readdressed the wavelength calibration. We are confident that the accuracy of the wavelengths is at least $0.02 \AA$. We also think that the oscillator strengths of these lines are correct because, together with other Fe vII lines, they are rather prominent in spectra of H-rich central stars of planetary nebulae (e.g. Rauch et al. 2007). Either way, the simultaneous fit of the detected Fe vII and Fe VIII lines independently confirms the validity of $T_{\text {eff }}$ and $\log g$ derived in earlier work.

In Table 2 we list the iron lines newly detected in PG 1159-035, together with lines from an Ne vi multiplet (NIST ${ }^{2}$ wavelengths) that we have discovered in the HST/STIS spectrum during the present analysis. This table complements the UV line list presented by Jahn et al. (2007).

In Table 3 we summarise the photospheric parameters of PG 1159-035 from spectroscopic analyses and derived quantities. In comparison to Jahn et al. (2007), the table is improved for both the $\mathrm{Fe}$ abundance and the re-determination of mass,

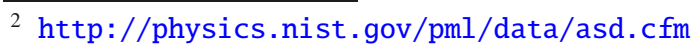

Table 2. New Fe viI, Fe viII, and Ne vi lines detected in PG 1159-035.

\begin{tabular}{|c|c|c|}
\hline Wavelength / Å & Ion & Transition \\
\hline 1006.09 & $\mathrm{Fe}$ VIII & $4 \mathrm{~s}^{2} \mathrm{~S}_{1 / 2}-3 \mathrm{~d}^{2}{ }^{2} \mathrm{P}_{1 / 2}^{\mathrm{o}}$ \\
\hline 1062.44 & Fe VIII & $4 \mathrm{~s}^{2} \mathrm{~S}_{1 / 2}-4 \mathrm{p}{ }^{2} \mathrm{P}_{3 / 2}^{\mathrm{o}}$ \\
\hline 1073.95 & Fe viI & $4 \mathrm{~s}^{1} \mathrm{D}-4 \mathrm{p}^{1} \mathrm{P}^{\mathrm{o}}$ \\
\hline 1095.34 & Fe vII & $4 s^{3} D_{3}-4 p^{3} P_{2}^{o}$ \\
\hline 1117.58 & Fe viI & $4 \mathrm{~s}^{1} \mathrm{D}-4 \mathrm{p}^{1} \mathrm{~F}^{\mathrm{o}}$ \\
\hline 1125.49 & Fe viII & $4 \mathrm{~s}^{2} \mathrm{~S}_{1 / 2}-4 \mathrm{p}{ }^{2} \mathrm{P}_{1 / 2}^{\mathrm{o}}$ \\
\hline 1141.43 & Fe viI & $4 s^{3} D_{3}-4 p^{3} F_{4}^{o}$ \\
\hline 1148.22 & Fe vIII & $4 \mathrm{~s}^{2} \mathrm{~S}_{1 / 2}-3 \mathrm{~d}^{2}{ }^{2} \mathrm{P}_{3 / 2}^{\mathrm{o}}$ \\
\hline 1154.99 & Fe viI & $4 s^{3} D_{2}-4 p^{3} F_{3}^{o^{\prime}}$ \\
\hline 1163.88 & $\mathrm{Fe}$ VII & $4 s^{3} D_{2}-4 p^{3} D_{3}^{o}$ \\
\hline 1166.17 & Fe vII & $4 s^{3} \mathrm{D}_{1}-4 \mathrm{p}^{3} \mathrm{~F}_{2}^{\mathrm{o}}$ \\
\hline 1180.82 & Fe vII & $4 s^{3} D_{3}-4 p^{3} D_{3}^{o}$ \\
\hline 1226.65 & Fe vII & $4 s^{3} D_{3}-4 p^{3} D_{2}^{o}$ \\
\hline 1239.69 & Fe viI & $4 s^{3} D_{1}-4 p^{3} D_{1}^{o}$ \\
\hline 1332.38 & Fe viI & $4 s^{1} D-4 p{ }^{1} D^{o}$ \\
\hline 1645.06 & $\mathrm{Ne}$ VI & $3 s^{4} \mathrm{P}_{1 / 2}^{\mathrm{o}}-3 \mathrm{p}^{4} \mathrm{P}_{3 / 2}$ \\
\hline 1645.59 & $\mathrm{Ne}$ vI & $3 s^{4} P_{3 / 2}^{o}-3 p p^{4} P_{5 / 2}$ \\
\hline 1654.01 & $\mathrm{Ne}$ VI & $3 s^{4} P_{1 / 2}^{o}-3 p^{4} P_{1 / 2}$ \\
\hline 1657.16 & $\mathrm{Ne}$ VI & $3 s^{4} P_{3 / 2}^{o}-3 p p^{4} P_{3 / 2}$ \\
\hline 1666.24 & $\mathrm{Ne}$ VI & $3 s^{4} P_{3 / 2}^{o}-3 p^{4} P_{1 / 2}$ \\
\hline 1667.82 & $\mathrm{Ne}$ vI & $3 s^{4} P_{5 / 2}^{o}-3 p^{4} P_{5 / 2}$ \\
\hline 1679.67 & $\mathrm{Ne}$ VI & $3 s^{4} P_{5 / 2}^{o}-3 p^{4} P_{3 / 2}$ \\
\hline
\end{tabular}

Notes. This table augments the UV line list of Jahn et al. (2007), their Table 2.

luminosity, and distance based on more realistic evolutionary tracks of Miller Bertolami \& Althaus (2006). 
K. Werner et al.: Iron abundance in PG 1159-035 and related objects

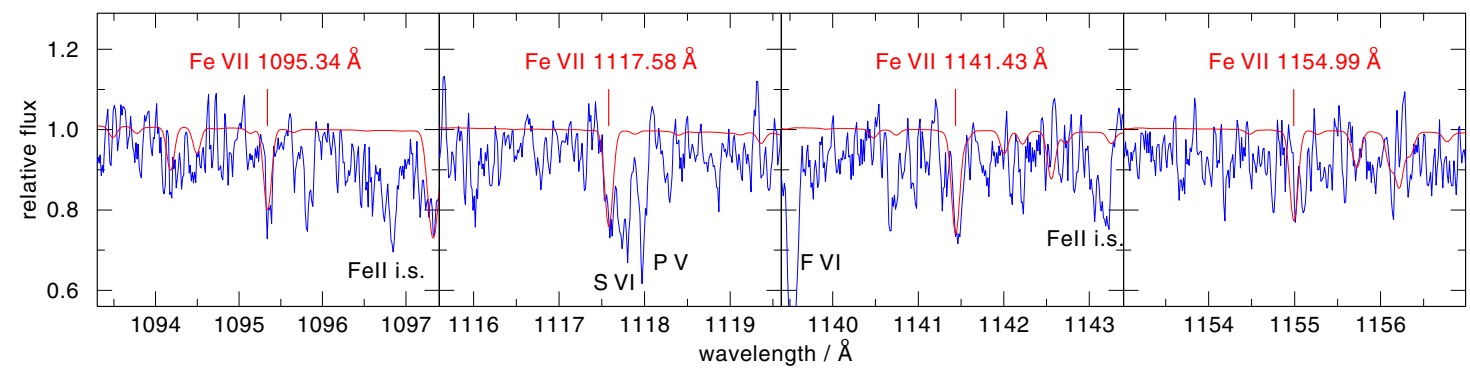

Fig. 6. Fe vII lines in PG 1424+535. Overplotted is a model with solar iron abundance (model parameters: $T_{\text {eff }}=110000 \mathrm{~K}, \log g=7, \mathrm{He} / \mathrm{C} / \mathrm{O} / \mathrm{Ne}=$ $0.49 / 0.43 / 0.06 / 0.02)$.

Table 3. Parameters of PG 1159-035.

\begin{tabular}{lccc}
\hline \hline Parameter & Result & $\begin{array}{c}\text { Abundances } \\
\text { (solar units) }\end{array}$ & Ref. \\
\hline$T_{\text {eff }} / \mathrm{K}$ & $140000 \pm 5000$ & & $(1),(2)$ \\
$\log g / \mathrm{cm} \mathrm{s}^{-2}$ & $7.0 \pm 0.5$ & & $(1),(2)$ \\
$\mathrm{H}$ & $\leq 0.02$ & $\leq 0.027$ & $(2)$ \\
$\mathrm{He}$ & 0.33 & 1.3 & $(2)$ \\
$\mathrm{C}$ & 0.48 & 203 & $(2)$ \\
$\mathrm{N}$ & 0.001 & 1.4 & $(2)$ \\
$\mathrm{O}$ & 0.17 & 30 & $(2)$ \\
$\mathrm{F}$ & $3.2 \times 10^{-6}$ & 6.3 & $(2)$ \\
$\mathrm{Ne}$ & 0.02 & 16 & $(2)$ \\
$\mathrm{Si}$ & $3.6 \times 10^{-4}$ & 0.54 & $(2)$ \\
$\mathrm{P}$ & $\leq 6.4 \times 10^{-6}$ & $\leq 1.1$ & $(2)$ \\
$\mathrm{S}$ & $5.0 \times 10^{-6}$ & 0.016 & $(2)$ \\
$\mathrm{Fe}$ & $1.3 \times 10^{-3}$ & 1.0 & $(1)$ \\
$M / M_{\odot}$ & $0.536_{-0.010}^{+0.068}$ & & $(1)$ \\
$\log L / L_{\odot}$ & $2.58_{-0.29}^{+0.29}$ & & $(1)$ \\
$d / \mathrm{kpc}$ & $0.750_{-0.585}^{+0.334}$ & & $(1)$ \\
\hline
\end{tabular}

Notes. Element abundances are given in mass fractions (2nd column) and relative to solar abundances (Asplund et al. (2009) values; 3rd column).

References. (1) this work, (2) Jahn et al. (2007) and references therein.

\section{2. $P G 1144+005, P G 1520+525, P G 1424+535$}

The two other PG 1159 stars in which we found Fe vIII lines are PG $1144+005\left(T_{\text {eff }}=150000 \mathrm{~K}, \log g=6.5\right)$ and PG $1520+525$ $\left(T_{\text {eff }}=150000 \mathrm{~K}, \log g=7.5\right)$. The lines are fitted with profiles from models with solar iron abundance (Fig. 2). We do not detect Fe viI lines in these stars, because they are hotter than PG 1159-035 so that we expect weaker lines, and because the $\mathrm{S} / \mathrm{N}$ of the available FUSE spectra is worse. The abundances of the models shown in Fig. 2 are $\mathrm{He} / \mathrm{C} / \mathrm{O} / \mathrm{Ne}=0.43 / 0.38 / 0.17 / 0.02$ for PG $1520+525$ and $0.38 / 0.58 / 0.02 / 0.02$ for PG $1144+005$.

The fourth PG 1159 star considered in the present study, PG $1424+535$, is too cool to exhibit Fe vIII lines. The star is interesting because an iron deficiency of at least 1 dex was concluded from the claimed absence of Fe vII (Reiff et al. 2008). Similar to the case of PG 1159-035, we again addressed this question and found that Fe viI lines are present after all. In Fig. 6 we show a selection of Fe vII lines from the FUSE spectrum compared to a solar iron abundance model. The match is very good.

\section{Summary and conclusions}

Our analysis of Fe viI and Fe vIII lines in the FUSE spectra of four PG 1159 stars results in solar iron abundances. Recent work on five hotter PG 1159 stars exhibiting Fe x lines (Werner et al. 2010) arrived at the same result. This set of nine stars comprises four objects, which previously were supposedly iron deficient: PG 1159-035, PG 1520+525, PG 1424+535, K 1-16 (Miksa et al. 2002; Jahn et al. 2007). The reason for this contrary result is twofold: an underestimation of $T_{\text {eff }}$ in the case of K 1-16 (Werner et al. 2010) and problems with the identification of the inherently weak lines from Fe vII as described in the present work. Fe viI was the only relevant ionisation stage with accurately known line positions at that time when earlier analyses were performed.

There are still two objects left with seemingly strong iron deficiency. These are the hybrid-PG 1159 star NGC 7094 and the [WC]-PG 1159 transition object Abell 78 (Miksa et al. 2002). In both stars we have discovered strong Fe vIII lines (Fig. 1). They are much broader than predicted from our static models. The reason is most probably that the lines form in the stellar wind of these low-gravity (i.e. high-luminosity) central stars. The same mechanism could hamper the detection of weak Fe vII lines, whose apparent absence the assertion of Fe deficiency was based on. Because of the prominent Fe vIII lines, we may speculate that the iron abundance in these objects is about solar, too, but a detailed analysis with expanding model atmospheres is required. Our results ease the problem of explaining the previously believed extreme iron deficiency with stellar evolution models, which do not predict such large Fe depletions by neutron captures in the intershell region of AGB stars.

Two of the objects investigated in this study (PG 1159-035 and PG $1520+525$ ) have rather similar parameters and they can be regarded as a fixed point for the blue edge of the GW Vir instability strip, at least for a particular chemical envelope composition. Within error limits, they have the same atmospheric abundance pattern (in particular the Fe abundance) and, thus, differences in the pulsation driving behaviour should only result from differences in $T_{\text {eff }}$ and $\log g$.

Our correct match of the Fe vII/Fe vIII ionisation balance corroborates the previously determined parameters for the pulsator PG 1159-035: $T_{\text {eff }}=140000 \mathrm{~K}, \log g=7.0$. The non-pulsator PG $1520+525$ has $T_{\text {eff }}=150000 \mathrm{~K}, \log g=7.5$. These parameters are confirmed by an analysis of its Chandra X-ray spectrum (Adamczak et al., in prep.).

Acknowledgements. T.R. is supported by the German Aerospace Centre (DLR) under grant 05 OR 0806. Some of the data presented in this paper were obtained from the Multimission Archive at the Space Telescope Science Institute (MAST). STScI is operated by the Association of Universities for Research in Astronomy, Inc., under NASA contract NAS5-26555. Support for MAST 
for non-HST data is provided by the NASA Office of Space Science via grant NNX09AF08G and by other grants and contracts.

\section{References}

Asplund, M., Grevesse, N., Sauval, A. J., \& Scott, P. 2009, ARA\&A, 47, 481 Costa, J. E. S., Kepler, S. O., Winget, D. E., et al. 2008, A\&A, 477, 627

Dreizler, S., \& Werner, K. 1996, A\&A, 314, 217

Hoffmann, A. I. D., Traulsen, I., Werner, K., et al. 2005, ASP Conf. Ser., 334, 321

Jahn, D., Rauch, T., Reiff, E., et al. 2007, A\&A, 462, 281

Kurucz, R. L. 2009, in Recent Directions in Astrophysical Quantitative Spectroscopy and Radiation Hydrodynamics, ed. I. Hubeny et al., AIP Conf. Proc., 1171, 43

Landi, E., \& Young, P. R. 2010, ApJ, 713, 205
McGraw, J. T., Starrfield, S. G., Liebert, J., \& Green, R. 1979, in White Dwarfs and Variable Degenerate Stars, ed. H. M. van Horn, \& V. Weidemann, Rochester, IAU Coll., 53, 377

Miksa, S., Deetjen, J. L., Dreizler, S., et al. 2002, A\&A, 389, 953

Miller Bertolami, M. M., \& Althaus, L. G. 2006, A\&A, 454, 845

Quirion, P.-O., Fontaine, G., \& Brassard, P. 2007, ApJS, 171, 219

Rauch, T., Ziegler, M., Werner, K., et al. 2007, A\&A, 470, 317

Reiff, E., Werner, K., Rauch, T., Koesterke, L., \& Kruk, J. W. 2008, in HydrogenDeficient Stars, ed. K. Werner, \& T. Rauch, ASP Conf. Ser., 391, 121

Starrfield, S., Cox, A. N., Kidman, R. B., \& Pesnell, W. D. 1984, ApJ, 281, 800 Wassermann, D., Werner, K., Rauch, T., \& Kruk, J. W. 2010, A\&A, 524, A9

Werner, K., \& Herwig, F. 2006, PASP, 118, 183

Werner, K., Rauch, T., Reiff, E., Kruk, J. W., \& Napiwotzki, R. 2004, A\&A, 427, 685

Werner, K., Rauch, T., \& Kruk, J. W. 2010, ApJ, 719, L32

Wesemael, F., Green, R. F., \& Liebert, J. 1985, ApJS, 58, 379 